\title{
On the solution of the random link matching problems
}

\author{
M. Mézard $\left({ }^{1}\right)$ and G. Parisi ( $\left.{ }^{2}\right)$ \\ ( $\left.{ }^{1}\right)$ Laboratoire de Physique Théorique de l'Ecole Normale Supérieure (*), 24, rue Lhomond, 75231 Paris \\ Cedex 05, France \\ $\left(^{2}\right)$ Universita' di Roma II Tor Vergata and INFN Roma, Italy
}

(Reçu le 29 avril 1987, accepté le 27 mai 1987)

\begin{abstract}
Résumé. - Nous montrons que la solution symétrique dans les répliques du problème d'appariement (bipartite ou pas) dans lequel les distances sont des variables aléatoires indépendantes est stable. Nous calculons les fluctuations et obtenons les corrections d'ordre $1 / N$ pour la longueur de l'appariement optimal dans un échantillon générique.
\end{abstract}

\begin{abstract}
We show that the replica symmetric solution of the matching problem (bipartite or not) with independent random distances is stable. We compute the fluctuations and get the $O(1 / N)$ corrections to the length of the optimal matching in a generic sample.
\end{abstract}

\section{Introduction.}

It has been recognized recently that the analytic methods developed in the study of the mean field theory of spin glasses [1] can be applied to some problems with finite range interactions : optimization problems [2] such as the random link matching [3, 4] and travelling salesman problem $[5,10,11]$ or the bipartitioning of random graphs with finite valence [7], or more conventional statistical physics problems such as randomly diluted magnets [6-8].

Tentative solutions to these problems have been proposed within the assumption of replica symmetry [3, 5-7]. Even within this simple hypothesis these problems require the introduction of an infinite set of order parameters. The reason for this is easily understood from the cavity method $[1,9]$. Because of the finite range of the interactions, the distribution of local fields is no longer a Gaussian (as is the case when the fields due to an infinity of neighbours add up), but a complicated function, the description of which requires an infinity of parameters (e.g. all the moments of the distribution).

In this type of situation the only attempt to study the stability of the replica symmetric solution, so far, has been performed in the case of diluted spin

(*) Laboratoire Propre du Centre National de la Recherche Scientifique, associé à l'Ecole Normale Supérieure et à l'Université de Paris Sud. glasses, in an expansion near the paramagnetic-spin glass transition temperature, where only the first two order parameters are relevant [8].

In this note we study the stability of the replica symmetric solution in the matching problem (whether bipartite or not) in which the distances are independent random variables [3]. Specializing for definiteness to the case in which these variables are uniformly distributed on the interval $[0,1]$ (this will be called hereafter the case of «flat distances »), we demonstrate that the replica symmetric solution is stable in both cases (matching and assignment also called bipartite matching), at any temperature, and therefore taking all the order parameters into account.

The stability can be studied either with the replica method or with the cavity method. We shall use the former and provide the generalization of the De Almeida-Thouless computation [12] to finite range problems. We have chosen this presentation because the replica method enables one to compute the finite $N$ corrections to the free energy in a very efficient and compact way.

In the next section we present the formalism for studying the stability in the replica method. The presentation is done on the example of the matching. In the case of flat independent distances we prove the stability at any temperature and we compute the $\mathrm{O}(1 / N)$ corrections to the ground state energy (= length of the optimal matching). 
In section 3 we perform the stability analysis for the problem of assignment (bipartite matching). The motivation for this study is that there exist extremely powerful algorithms for this problem [13, 14], so that we obtained good numerical data [4] to which our predictions can be confronted. The study of stability is more subtle than in the matching because of the existence of a continuous symmetry (in replica space) which induces a number of Goldstone modes. Taking care of them we show the stability and compute the $\mathrm{O}(1 / N)$ corrections to the optimal assignment in the case of flat distances. The result (52) fits the numerical data.

Section 4 contains a summary and some remarks.

\section{The matching problem.}

In the matching problem one is given $2 N$ points $i=1, \ldots, 2 N$ and the set of distances $d_{i j}=d_{i i}$. We suppose that the $d$ 's are identically distributed independent random variables and for definiteness we shall often specialize to the case of flat distances where the $d$ 's are uniformly distributed on the interval $[0,1]$. (This is the $r=0$ case of [3] ; any other reasonable distribution can be studied as well [3].) A configuration $\mathcal{C}$ is a set of $N$ links which connect pairwise all the points. Its energy $E(\mathcal{C})$ is the total length of the links. One introduces a partition function :

$$
Z=\sum_{\mathrm{e}} \mathrm{e}^{-\beta N E(\mathrm{e})}
$$

where the scaling of the inverse temperature $\beta$ with $N$ has been chosen in order to ensure the existence of a good thermodynamic limit at fixed $\beta[10]$.

In [3] we used the replica method to compute the $n$ 'th power of the partition function. There appears, as natural order parameters, a set of $2^{n}-1$ variables $Q_{a_{1} a_{2}} \cdots a_{p}$ where $1 \leqslant a_{1} \leqslant a_{2}<\ldots<a_{p} \leqslant n$ and $1 \leqslant$ $p \leqslant n$. In order to lighten the notations we shall sometimes use a single index $\alpha=1, \ldots, 2^{n}-1$ to denote the set of numbers $p ; a_{1}, a_{2}, \ldots, a_{p}$. The corresponding value of $p$ will be denoted $p(\alpha)$. The result of [3] is :

$$
\begin{aligned}
& \bar{Z}^{n N} \rightarrow \infty \int \prod_{\alpha} \frac{\mathrm{d} Q_{\alpha}}{\sqrt{2 \pi g_{p(\alpha)} / N}} \exp -\frac{N}{2} S[Q] \times \\
& \times \exp -\frac{1}{4} \sum_{\alpha, \gamma}{ }^{\prime} g_{p(\alpha)} g_{p(\gamma)}\left[\frac{Q_{\alpha \cup \gamma}}{2 g_{p(\alpha)+p(\gamma)}}\right]^{2}
\end{aligned}
$$

where :

$$
S[Q]=\sum_{\alpha} \frac{1}{g_{p(\alpha)}} Q_{\alpha}^{2}-4 \log z[Q]
$$

$$
\begin{aligned}
& z[Q]=\left(\prod_{a=1}^{n} \int_{0}^{2 \pi} \frac{\mathrm{d} \lambda^{a}}{2 \pi} \mathrm{e}^{i \lambda^{a}}\right) \times \\
& \quad \times \exp \left(\sum_{p=1}^{n} \sum_{a_{1}<\cdots<a_{p}} Q_{a_{1} \cdots a_{p}} \mathrm{e}^{-i\left(\lambda_{a_{1}}+\cdots+\lambda_{a_{p}}\right)}\right)
\end{aligned}
$$

and $g_{p}$ is a function of the temperature which depends on the distribution of couplings $[3,10]$. For flat distances $g_{p}=1 /(p \beta)$. The last term in (2) was omitted in [3] since it gives an $\mathrm{O}(1 / N)$ correction to the free energy. The $\sum_{\alpha, \gamma}{ }^{\prime}$ means a sum over $p, q$ from 1 to $n$ and $a_{1}<\cdots<a_{p}, b_{1}<\cdots<b_{q}$, such that the $a$ 's and the $b$ 's are all different from each other. $Q_{\alpha \cup \gamma}$ is the corresponding variable $Q_{a_{1} \ldots a_{p} b_{1} \ldots b_{q}}$

The leading term in (2) is obtained by the saddle point method. A solution $Q_{\alpha}^{\text {s.p. }}$ for the saddle point equations :

$$
Q_{\alpha}=2 g_{p(\alpha)} \frac{\partial \log z}{\partial Q_{\alpha}}
$$

was given in [3] within the hypothesis of replica symmetry :

$$
Q_{a_{1} \ldots a_{p}}^{\text {s.p. }}=Q_{p}^{\text {s.p. }}
$$

independently of the values of $a_{1} \ldots a_{p}$.

In order to study the stability we introduce the fluctuations of the $Q$ 's around their saddle point values :

$$
Q_{\alpha}=Q_{p(\alpha)}^{\text {s.p. }}+\tilde{Q}_{\alpha}
$$

and expand the action $S[Q]$ in powers of $\tilde{Q}$ up to second order. The replica symmetric saddle point (5) will be stable if and only if the quadratic form in the $\tilde{Q}$ has only nonnegative eigenvalues in the $n \rightarrow 0$ limit [12]. if this is the case one can rewrite (2) as :

$$
\bar{Z}^{n} \stackrel{\substack{N \rightarrow \infty \\ n \rightarrow 0}}{\sim} \mathrm{e}^{-n N \beta\left(F^{\text {s.p. }}+\Delta F^{1}+\Delta F^{2}\right)}
$$

where $F^{\text {s.p. }}$ is the saddle point contribution to the free energy, already computed in [3], $\Delta F^{1}$ is the $\mathrm{O}(1 / N)$ contribution coming from the last term in (2) :

$\Delta F^{1}=\lim _{n \rightarrow 0} \frac{1}{4 n N \beta} \sum_{\alpha, \gamma}^{\prime} g_{p(\alpha)} g_{p(\gamma)}\left(\frac{Q_{p}^{\text {s.p. }}(\alpha)+p(\gamma)}{2 g_{p(\alpha)+p(\gamma)}}\right)^{2}$

and $\Delta F^{2}$ is the contribution from the Gaussian fluctuations around the saddle point :

$$
\begin{aligned}
\Delta F^{2}=\lim _{n \rightarrow 0} \frac{-1}{n N \beta} \log \int & \prod_{\alpha} \frac{\mathrm{d} \tilde{Q}_{\alpha}}{\sqrt{2 \pi / N}} \cdot \exp -\frac{N}{2} \sum_{\alpha} \tilde{Q}_{\alpha}^{2} \times \exp N \sum_{\alpha, \gamma}{ }^{\prime} \tilde{Q}_{\alpha} \tilde{Q}_{\gamma} \frac{Q_{p(\alpha)+p(\gamma)}^{\text {s.p. }}}{2 g_{p(\alpha)+p(\gamma)}} \sqrt{g_{p(\alpha)} g_{p(\gamma)}} \times \\
& \times \exp -N \sum_{\alpha} \sum_{\gamma} \tilde{Q}_{\alpha} \tilde{Q}_{\alpha}\left(Q_{p(\alpha)}^{\text {s.p. }} / 2 \sqrt{\left.g_{p(\alpha)}\right)}\right)\left(Q_{p(\gamma)}^{\text {s.p. }} / 2 \sqrt{g_{p(\gamma)}}\right.
\end{aligned}
$$


Knowing the saddle point solution $Q^{\text {s.p. }}$ [3], the evaluation of $\Delta F^{1}$ is an exercice of combinatorics and computation of integrals which we sketch in the appendix. The result is :

$$
\begin{aligned}
\Delta F^{1} & =-\frac{1}{N}(\zeta(3) / 8) \\
& =-\frac{1}{N}(0.150257 \ldots) .
\end{aligned}
$$

In order to compute the fluctuations (9) we follow the strategy of [12] and diagonalize the quadratic form inside subspaces of increasing size. We must find the $2^{n}-1$ eigenvectors $\tilde{Q}_{\alpha}$ and eigenvalues $\lambda$ which satisfy :

$$
\begin{array}{r}
\tilde{Q}_{a_{1} \ldots a_{p}}-2 \sum_{q=1}^{n-p} \sqrt{g_{p} g_{q}} \frac{Q_{p+q}^{\text {s.p. }}}{2 g_{p+q}} \sum_{\substack{b_{1}<\cdots<b_{q} \\
\{a\} \cap\{b\}=\varnothing}} \times \\
\times \tilde{Q}_{b_{1} \ldots b_{q}}=\lambda \tilde{Q}_{a_{1} \ldots a_{q}} .
\end{array}
$$

(It turns out that the last term in (9) always gives a zero contribution when $n \rightarrow 0$, as is seen by inspection in each of the families of eigenmodes studied below.)

We begin with the longitudinal modes:

$$
\tilde{Q}_{a_{1} \ldots a_{p}}=c_{p} .
$$

The $c_{p}$ 's satisfy the set of equations: ( $p=$ $1,2, \ldots, \infty)$ :

$$
\begin{aligned}
& \frac{\Gamma(n)}{\Gamma(n-p+1) \Gamma(p+1)}\left[(\lambda-1) c_{p}+\right. \\
& +2 \sum_{q=1}^{\infty} \frac{Q_{p+q}^{\text {s.p. }}}{2 g_{p+q}} \sqrt{g_{p} g_{q}} \\
& \left.\quad \times \frac{\Gamma(n-p+1)}{\Gamma(n-p-q+1) \Gamma(q+1)} c_{q}\right]=0
\end{aligned}
$$

where the $\Gamma$ functions cancel the terms where $p$ or $p+q$ are larger than $n$. Taking the $n \rightarrow 0$ limit in
(13), we find an infinite set of eigenvalues/eigenvectors which are those of the infinite dimensional matrix $M^{(1)}$, the first element of an infinite family of matrices $M^{(k)}$ which will be useful in the following, defined as :

$$
\begin{aligned}
M_{p, q}^{(k)}=\delta_{p, q}-2(-1)^{q+k} & \frac{(p+q+2 k-1) !}{(p+2 k-1) ! q !} \times \\
& \times g_{q+k} \frac{Q_{p+q+2 k}^{\text {s.p. }}}{2 g_{p+q+2 k}}
\end{aligned}
$$

$(p, q=0,1, \ldots, \infty)$.

(In order to obtain $M^{(1)}$ from (13), we have shifted $p$ and $q$ by 1 and multiplied each matrix element by $\sqrt{g_{q+1} / g_{p+1}}$, which does not change the spectrum). We will show hereafter that in general the eigenvalues in the $k^{\prime}$-th family will be those of the matrix $M^{(k)}$.

After the longitudinal modes we introduce a family of modes in which one replica is distinguished, let us say replica 1 (see [12]). Thus :

$$
\tilde{Q}_{a_{1} \ldots a_{p}}= \begin{cases}d_{p} & \text { if } 1 \in\left\{a_{1}, \ldots, a_{p}\right\} \\ e_{p} & \text { if not }\end{cases}
$$

Orthogonalization of this vector with the longitudinal eigenvector gives :

$$
p d_{p}+(n-p) e_{p}=0 .
$$

Therefore in the limit $n \rightarrow 0$ we have $d_{p}=e_{p}$ and the spectrum is the same as in the previous case. On the whole we have found so far that in the $n \rightarrow 0$ limit all the eigenvalues of $M^{(1)}$ belong to the spectrum of fluctuations, each with a degeneracy equal to $n$ (the number of ways one can choose the distinguished replica).

Let us turn to the second family of transverse modes, which has two distinguished replicas, say 1 and 2. They have $Q_{a}=0$ and, for $p>2$ :

$$
Q_{a_{1} \ldots a_{p}}=\left\{\begin{array}{l}
f_{p} \text { if } 1 \in\left\{a_{1}, \ldots, a_{p}\right\} \text { and } 2 \in\left\{a_{1}, \ldots, a_{p}\right\} \\
g_{p} \text { if } 1 \text { or } 2, \text { but not both, belongs to }\left\{a_{1}, \ldots, a_{p}\right\} \\
h_{p} \text { if neither } 1 \text { nor } 2 \text { belongs to }\left\{a_{1}, \ldots, a_{p}\right\}
\end{array}\right.
$$

Orthogonalization with respect to the previous eigenvectors gives in the $n \rightarrow 0$ the limit :

$$
\frac{f_{p}}{p(p+1)}=\frac{g_{p}}{(p-1)(p+1)}=\frac{h_{p}}{(p-1) p} .
$$

Using (11), (17) and (18) one finds, after shifting the indices $p$ and $q$ by two, that the eigenvalues of this second family are nothing but those of the matrix $M^{(2)}$. The degeneracy of this second family is [12] :

$$
C_{n}^{2}-C_{n}^{1}=n(n-3) / 2^{n \rightarrow 0} \sim\left(-\frac{3}{2}\right) .
$$

We shall not write down in detail the generaliza- tion to the $k^{\prime}$-th transverse family which has $k$ distinguished replicas. Let us just mention the form of the eigenvectors :

$$
Q_{a_{1} \ldots a_{p}}= \begin{cases}0 & \text { if } p<k \\ d_{p}^{i} & \text { if } a_{1}, \ldots, a_{p} \\ & \text { contains } k+1-i \\ & \text { distinguished repli- } \\ & \text { cas, where } i \text { goes } \\ & \text { from one to } k+1\end{cases}
$$

and the generalization of (18) obtained from orthogonalization with respect to previous families : 


$$
\begin{aligned}
\frac{d_{p}^{1}}{p(p+1) \cdots(p+k-2)} & =\frac{d_{p}^{2}}{(p-k+1)(p+1) \cdots(p+k-2)}= \\
= & \frac{d_{p}^{3}}{(p-k+1)(p-k+2)(p+2) \cdots(p+k-2)}=\cdots=\frac{d_{p}^{k+1}}{(p-k+1) \cdots p} .
\end{aligned}
$$

Plugging this into (11), one finds that the spectrum in the $k^{\prime}$-th family is nothing but that of $M^{(k)}$. Its degeneracy is :

$$
x_{k}=C_{n}^{k}-C_{n}^{k-1} \stackrel{n \rightarrow 0}{\sim} n(-1)^{k-1} \frac{2 k-1}{k(k-1)} .
$$

Stability of the replica symmetric solution is equivalent to having all the eigenvalues of all the matrices $M^{(k)}(k=1, \ldots, \infty)$ nonnegative, and it this solution is stable, the contribution of the fluctuations to the $O(1 / N)$ correction is :

$$
\begin{aligned}
\Delta F^{2} & =\frac{1}{2 N \beta} \times\left[\log \operatorname{det} M^{(1)}+\right. \\
& \left.+\sum_{k=2}^{\infty} \frac{(-1)^{k-1}(2 k-1)}{k(k-1)} \log \operatorname{det} M^{(k)}\right] .
\end{aligned}
$$

At this stage we have taken care of the specific problems of diagonalizing $0 \times 0$ matrices, and we have reduced the problem to the evaluation of the spectra of all the matrices $M^{(k)}$ defined in (14), $k=1, \ldots, \infty-$ an infinite set of infinite dimensional matrices. We shall now use the special properties of the replica symmetric solution of the matching problem [3]. As was noticed in [3], the $Q^{\text {s.p. by }}$ themselves are not very good order parameters (for the flat distances case they are not even well defined at low temperatures), and it is more convenient to introduce the order parameter function [3] :

$$
G(\ell)=\sum_{p=1}^{\infty}(-1)^{p-1} \frac{Q_{p}^{\text {s.p. }} \mathrm{e}^{\ell p}}{p !} .
$$

Using the saddle point equations (4) which read :

$$
Q_{p}^{\text {s.p. }}=2 g_{p} \int_{-\infty}^{+\infty} \mathrm{d} \ell \frac{\mathrm{e}^{\ell p}}{p !} \mathrm{e}^{-G(\ell)}
$$

the matrix $M^{(k)}$ can be written as :

$$
\begin{gathered}
M^{(k)}=\mathbb{1}-(-1)^{k} A^{(k)} \\
A_{p, q}^{(k)}=2(-1)^{q} \frac{g_{q+k}}{(p+2 k-1) ! q !} \times \\
\times \int \mathrm{d} \ell \mathrm{e}^{(2 k+p+q) \ell-G(\ell)} .
\end{gathered}
$$

Now it is natural to change bases: if $f_{p}$ is an eigenvector of $M^{(k)}$ with eigenvalue $\lambda$, the function :

$$
f(x)=\sum_{q=0}^{\infty} \frac{(-1)^{q}}{q !} g_{q+k} f_{q} \mathrm{e}^{(k+q) x-\frac{G(x)}{2}}
$$

is an eigenvalue of the operator $\hat{M}^{(k)}$ with the same eigenvalue, and reciprocally. $\hat{M}^{(k)}$ is defined as :

$$
\begin{gathered}
\hat{M}^{(k)}(x, y)=\delta(x-y)-(-1)^{k} \hat{A}^{(k)}(x, y) \\
\hat{A}^{(k)}(x, y)=2 \exp \left(-\frac{G(x)+G(y)}{2}+k(x+y)\right) \times \\
\times\left[\sum_{p=0}^{\infty} \frac{(-1)^{p} \mathrm{e}^{p(x+y)}}{p !(2 k+p-1) !} g_{p+k}\right] \cdot \quad(27)
\end{gathered}
$$

$\hat{M}^{(k)}$ is now a well defined operator : $G$ is the saddle point order parameter function which can be computed at any temperature and for any distribution of the distances [3]. We have diagonalized $\hat{M}^{(k)}$ in the case of flat distances, following a simple procedure : firstly we restrict the interval of variation of $x$ and $y$ from the whole plane to a square such that $\hat{A}^{(k)}(x, y)$ is very small outside the square. Then we discretize $\hat{A}^{(k)}$ on $P$ equidistant points in each direction $x$ and $y$. We are left with the diagonalization of a $P \times P$ matrix. It turns out that the relevant properties of the spectrum of $\hat{M}^{(k)}$ are reasonably independent of the cut-offs and of the number of points $P$ (for $P \sim 30$ to 90 ), at least for temperatures $T>0.3$. When one augments $P$, the new eigenvalues accumulate around $\lambda=1$, as they should since $\hat{A}^{(k)}$ is a regular operator. We have computed the spectra of $\hat{M}^{(k)}$ for $k$ between 1 and 12, and found all eigenvalues were nonnegative. The value of the minimal eigenvalue for each $k$ and $T$ between 0 and 0.7 , is plotted in figure 1.

The values of det $\left(\hat{M}^{(k)}\right)$ are also very stable with respect to the discretization procedure of $\hat{M}^{(k)}$. They share with the minimal eigenvalues a property which can be already seen in figure $1:$ For $k$ even and $k$ odd separately $(k \geqslant 2)$, det $\hat{A}^{(k)}$ is a function of $T \log k$ at low enough temperatures $(T \leqslant 0.7)$. This is seen on figure 2 where the crosses are the values of det $\hat{M}^{(k)}$, as functions of $T \log k$ for $k \geqslant 2:$ they fall onto universal curves, $f_{+}(T \log k)$ for $k$ even and $f_{-}(T \log k)$ for $k$ odd. Using (21), this property will allow us to compute the value $\Delta F^{(2)}$ at zero temperature $(\beta \rightarrow \infty)$. In this special limit the sums over $k$ in (22) (performed separately on $k$ even and $k$ odd) can be converted into an integral, and we find (leaving aside the term $k=1$ which does not contribute in the limit $T \rightarrow 0$ ) :

$$
\Delta F^{2} \stackrel{T \rightarrow 0}{=}-\frac{1}{2 N} \int_{0}^{\infty} \mathrm{d} u\left[\log f_{+}(u)-\log f_{-}(u)\right] \text {. }
$$



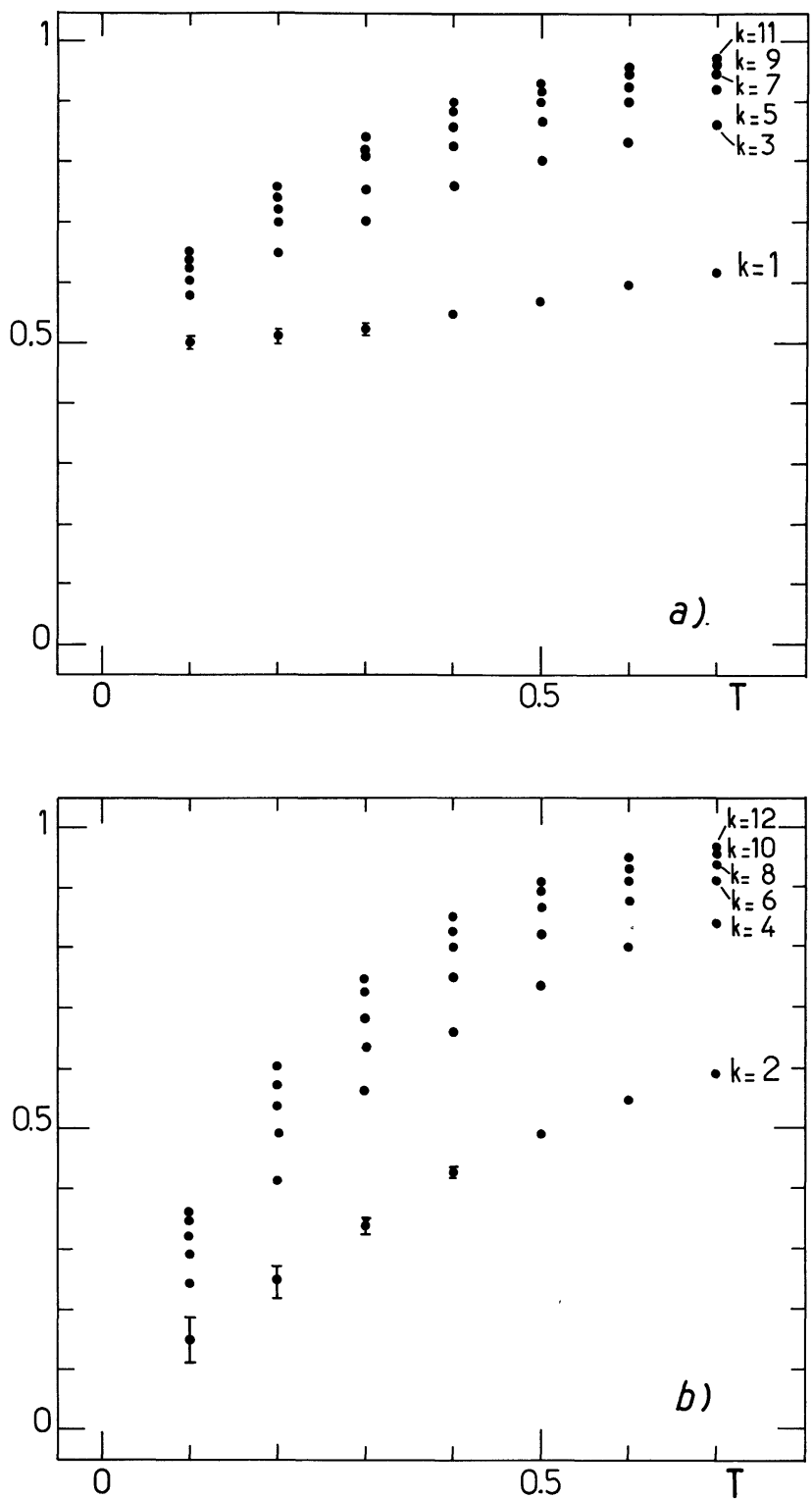

Fig. 1. - Plot of the minimal eigenvalue of the the matrix $M^{(k)}$ (stability matrix in the $k$-th family) as a function of temperature for $k=1$ to 12 . $k$ odd : figure $1 \mathrm{a} ; k$ even : figure $1 b$.

In order to compute these integrals, we have fitted the universal functions $f_{+}$and $f_{-}$by smooth functions; the curves in figure 2 are plots of the functions :

$$
\begin{aligned}
& f_{+}(u)=1-(1-0.42 u) \mathrm{e}^{-1.66 u} \\
& f_{-}(u)=1+0.99 u \mathrm{e}^{-2.61 u}
\end{aligned}
$$

(there are some rather large errors bars for the fit of $f_{-}$, especially at small values of $u$, but these have small effects on the value of $\Delta F^{2}$ ). The result for $\Delta F^{2}$ is :

$$
\Delta F^{2} \stackrel{T \rightarrow 0}{=} 1 / N(0.47 \pm 0.05)
$$

where the error is a subjective estimate of the
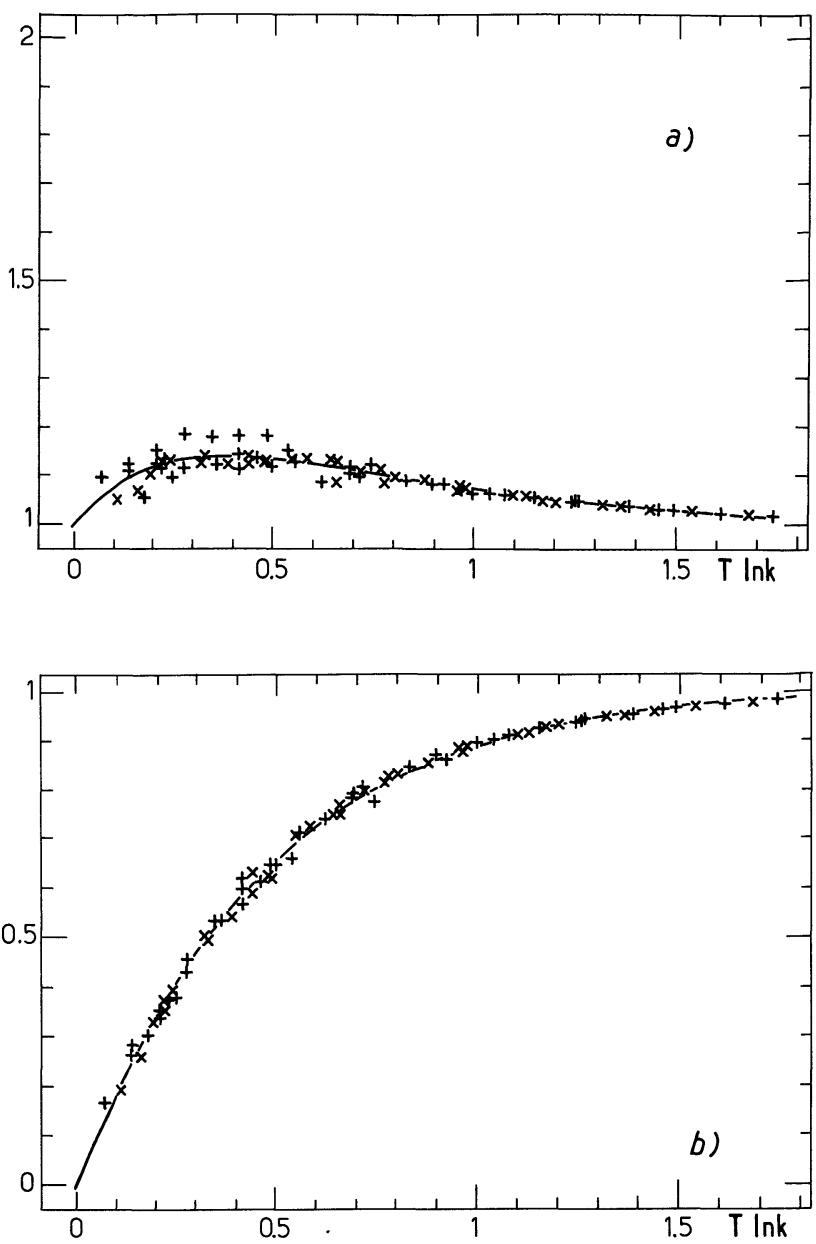

Fig. 2. - Values of the determinants det $\mathbf{M}^{(k, \varepsilon)}$ defined in (27) and (39) plotted versus $T \log k$, for $k=2$ to 12. Figure 2a contains det $M^{(k,-)} k$ even (denoted by + ) and $\operatorname{det} M^{(k,+)} k$ odd (denoted by $\times$ ). Figure $2 b$ contains $\operatorname{det} M^{(k,+)} k$ even $(+)$, det $M^{(k,-)} k$ odd $(\times)$. The lines are the fits (29).

uncertainties of the fit so that our final result for the length of the optimal matching is :

$$
L=\pi^{2} / 12+1 / N(0.32 \pm 0.05) .
$$

It would be interesting to confront this result with numerical simulations. We have done such a confrontation in another case, the assignment problem.

\section{The assignment problem.}

The problem of assignment (bipartite matching) is of interest since there exist particularly efficient algorithms to solve it. In this case one is given two sets - A and B - of $N$ points and one must find the shortest set of $N$ links which connect, pairwise, the points of $\mathrm{A}$ with the points of B. Taking, as before, the distances between the points in $\mathrm{A}$ and in $\mathrm{B}$ as independent random variables, and applying the same techniques as for the matching [3], we get, instead of (2) : 


$$
\bar{Z}^{n} \stackrel{N \rightarrow \infty}{\sim} \int \prod_{\alpha}\left(\frac{\mathrm{d} X_{\alpha} \mathrm{d} Y_{\alpha}}{2 \pi g_{p(\alpha)} / N}\right) \times \exp -N S[X, Y] \times \exp \left\{-\frac{1}{2} \sum_{\alpha, \gamma}{ }^{\prime} g_{p(\alpha)} g_{p(\gamma)} \frac{X_{\alpha}^{2}-Y_{\alpha}^{2}}{\left(g_{p(\alpha)+p(\gamma)}\right)^{2}}\right\}
$$

where :

$$
S[X, Y]=\sum_{\alpha} \frac{X_{\alpha}^{2}+Y_{\alpha}^{2}}{g_{p(\alpha)}}-\log z[X+i Y]-\log z[X-i Y]
$$

where the conventions are the same as in (2), and $z$ is the one-site partition function defined in (3). As before the leading term can be evaluated by a saddle point method. There exists a replica symmetric saddle point at :

$$
X_{a_{1} \ldots a_{p}}^{\text {s.p. }}=X_{p}^{\text {s.p. }} ; \quad Y_{a_{1} \ldots a_{p}}^{\text {s.p. }}=0
$$

and the $X^{\text {s.p. }}$ satisfy saddle point equations which are very similar to the $Q^{\text {s.p. }}$ of the matching. In fact one gets, for flat distances:

$$
X_{p}^{\text {s.p. }}(\beta)=Q_{p}^{\text {s.p. }}(\tilde{\beta}=2 \beta)
$$

so that the ground state energy of the bipartite matching is twice that of the matching [3].

As before, the $\mathrm{O}(1 / N)$ corrections to the free energy contain two terms. The $\Delta F^{1}$ coming from the last term in (32) is computed exactly as for the matching, and one gets :

$\Delta F^{1}$ (bipartite matching $)=16 . \Delta F^{1}$ (matching $)$.

Let us now compute the fluctuation term $\Delta F^{2}$. Expanding as before the action to quadratic order around the saddle point, we find :

$$
F^{2}=\lim _{n \rightarrow 0} \frac{-1}{n N \beta} \log (F(1) F(-1))
$$

with :

$$
\begin{aligned}
F(\varepsilon) & =\int \prod_{\alpha} \frac{\mathrm{d} X_{\alpha}}{\sqrt{2 \pi / N}} \exp -\frac{N}{2} \sum_{\alpha} X_{\alpha}^{2} \times \\
& \times \exp \frac{N \varepsilon}{2} \sum_{\alpha, \gamma}{ }^{\prime} X_{\alpha} X_{\gamma} \frac{\sqrt{g_{p(\alpha)} g_{p(\gamma)}}}{g_{p(\alpha)+p(\gamma)}} X_{p(\alpha)+p(\gamma)}^{\text {s.p. }} .
\end{aligned}
$$

Comparing with (9) (in (37) we have dropped the irrelevant disconnected contribution - last term of (9)), and using the relation (5) between the saddle point values of the order parameter in the matching and the assignment, we see that we are left with nearly the same stability condition as before: the eigenvalues of $M^{(k, \varepsilon)}$ defined by :

$$
M^{(k, \varepsilon)}=1-\varepsilon(-1)^{k} A^{(k)}
$$

(where $A^{(k)}$ is defined in (25)) must be nonnegative for any $\varepsilon= \pm 1$ and any $k=1,2, \ldots, \infty$. (The case $\varepsilon=+1$ is the case we studied in Sect. 2.) If this condition is satisfied :

$$
\begin{aligned}
& F(\varepsilon, \beta)=1-\frac{n}{2}\left[\log \operatorname{det} M^{(1, \varepsilon)}+\right. \\
& \left.\quad+\sum_{k=2}^{\infty} \frac{(-1)^{k-1}(2 k-1)}{k(k-1)} \log \operatorname{det} M^{(k, \varepsilon)}\right] .
\end{aligned}
$$

However we run into a problem because $\hat{M}^{(1,-1)}$ has a zero mode, corresponding to the eigenfunction $G^{\prime}(x) \exp \left(-\frac{G(x)}{2}\right)$. Being in the family $k=1$, it has a degeneracy equal to $n$. This is in fact normal because the action (33) possesses a symmetry under rotations in the $X, Y$ plane, in each replica independently. Precisely the action is left invariant under the transformations $(X, Y) \rightarrow\left(X^{\prime}, Y^{\prime}\right)$, where :

$$
\begin{aligned}
X_{a_{1} \ldots a_{p}}^{\prime} & +i Y_{a_{1} \ldots a_{p}}^{\prime}= \\
& =\left(X_{a_{1} \ldots a_{p}}+i Y_{a_{1} \ldots a_{p}}\right) \mathrm{e}^{i\left(\theta_{a_{1}}+\ldots+\theta_{a_{p}}\right)} .
\end{aligned}
$$

Thus the saddle point (34) is only one point of an $n$ dimensional orbit of saddle points parameterized by :

$$
\begin{gathered}
X_{a_{1} \ldots a_{p}}^{\text {s.p. }}+i Y_{a_{1} \ldots a_{p}}^{\text {s.p. }}=X_{p}^{\text {s.p. }} \mathrm{e}^{i\left(\theta_{a_{1}}+\ldots+\theta_{a_{p}}\right)} \\
0 \leqslant \theta_{a} \leqslant 2 \pi ; \quad 1 \leqslant a \leqslant n
\end{gathered}
$$

where the $X^{\text {s.p. }}$ is that of (34)-(35). The $n$ zero modes that we have found are the Goldstone modes of this invariance. In order to compute the fluctuations correctly, one must compute separately the volume of the orbit (42) and leave aside the zero modes corresponding to the motion along the orbit.

We begin by computing the fluctuations, without the zero mode of $M^{(1,-1)}$. Using the same method of discretization of the operators that we used for the matching, we have found that the previous scaling relation also holds with $\varepsilon=-1$ in the following sense. For any $k \geqslant 2$ :

$$
\begin{array}{ll}
\operatorname{det}\left(\mathbb{1}+\hat{A}^{(k)}\right)=f_{+}(T \log k) & T \ll 1 \\
\operatorname{det}\left(\mathbb{1}+\hat{A}^{(k)}\right)=f_{-}(T \log k) & T \ll 1 .
\end{array}
$$

This scaling is shown in figure 2. From (37), (40), (43) we have for $T \rightarrow 0$ :

$$
\begin{aligned}
\Delta F^{(2)} \stackrel{T \rightarrow 0}{\sim} & \frac{1}{2 N \beta} \sum_{k=2}^{\infty} \frac{(-1)^{k-1}(2 k-1)}{k(k-1)} \times \\
& \times\left[f_{+}(T \log k)+f_{-}(T \log k)\right] .
\end{aligned}
$$


Instead of having as in the matching (see (22)-(27)) a sum involving the even terms with the function $f_{+}$and the odd terms with $f_{-}$, all the terms contribute to both $f_{+}$and $f_{-}$. Because of the alternating signs in (44), the resulting contribution vanishes in the limit $\beta \rightarrow \infty$ :

$$
\Delta F^{2} \stackrel{\sim \rightarrow 0}{\sim} 0 \text {. }
$$

Finally, in order to complete the computation of the $O(1 / N)$ corrections in this bipartite case, we still must compute the volume of the hypersurface of saddle points. This orbit (42) is an $n$ dimensional hypersurface parameterized by the angles $\theta_{1}, \ldots, \theta_{n}$ in a space of dimension $2\left(2^{n}-1\right)$. In order to obtain the volume element $\mathrm{d} \tau / \mathrm{d} \theta_{1} \ldots \mathrm{d} \theta_{n}$ we introduce the metric:

$$
g_{a b}=\sum_{\alpha}\left[\frac{\partial X_{\alpha}^{\text {s.p. }}}{\partial \theta_{a}} \frac{\partial X_{\alpha}^{\text {s.p. }}}{\partial \theta_{b}}+\frac{\partial Y_{\alpha}^{\text {s.p. }}}{\partial \theta_{a}} \frac{\partial Y_{\alpha}^{\text {s.p. }}}{\partial \theta_{b}}\right]
$$

and $\mathrm{d} \tau / d \theta_{1} \ldots \mathrm{d} \theta_{n}$ is the square root of the determinant of $g$. It turns out that $g$ has a simple structure; all diagonal elements are equal to $g_{0}$ and all non diagonal elements are equal to $g_{1}$, with :

$$
\begin{aligned}
& g_{0}=\sum_{p=1}^{\infty} p\left(X_{p}^{\text {s.p. }}\right)^{2} \frac{\Gamma(n)}{\Gamma(n-p+1) \Gamma(p+1)} \\
& g_{1}=\sum_{p=1}^{\infty} p(p-1)\left(X_{p}^{\text {s.p. }}\right)^{2} \frac{\Gamma(n-1)}{\Gamma(n-p+1) \Gamma(p+1)} .
\end{aligned}
$$

The det $g$ is then easily evaluated :

$$
\sqrt{\operatorname{det} g} \stackrel{n \rightarrow 0}{\sim} 1+\frac{n}{2}\left[\frac{g_{1}}{g_{0}-g_{1}}+\log \left(g_{0}-g_{1}\right)\right] \text {. }
$$

This will give a new contribution $\Delta F^{3}$ to the free energy at order $1 / N$ :

$$
\Delta F^{3}=-\frac{1}{2 N \beta}\left[\frac{g_{1}}{g_{0}-g_{1}}+\log \left(g_{0}-g_{1}\right)\right] .
$$

Using the values (35) of the saddle point numbers $X_{p}^{\text {s.p. }}$ we find for $T \rightarrow 0$ and for flat distances:

$$
\begin{gathered}
g_{0} \stackrel{T \rightarrow 0}{\sim} \frac{\pi^{2}}{6} \\
g_{0}-g_{1} \stackrel{T \rightarrow 0}{\sim} T
\end{gathered}
$$

so that :

$$
\Delta F^{3 T \rightarrow 0}-\frac{1}{N} \frac{\pi^{2}}{12}
$$

We have thus found that the replica symmetric solution to the bipartite matching problem with independent random distances uniformly distributed on $[0,1]$ is stable. Collecting the contributions (36), (45) and (51), we find that the length of the optimal configuration, including the first corrections to $\mathrm{O}(1 / N)$, is :

$$
\begin{aligned}
L & =\frac{\pi^{2}}{6}-\frac{1}{N}\left(\frac{\pi^{2}}{12}+2 \zeta(3)\right)+\mathrm{O}\left(\frac{1}{N}\right)= \\
& =1.6449 \ldots-1 / N(3.2266 \ldots)+\mathrm{O}\left(\frac{1}{N}\right) .
\end{aligned}
$$

In figure 3 we reproduce a plot from [14] giving the average length of the optimal assignment for samples with $2 N$ between 100 and 800 . The straight line is the prediction (52). If fits the data.

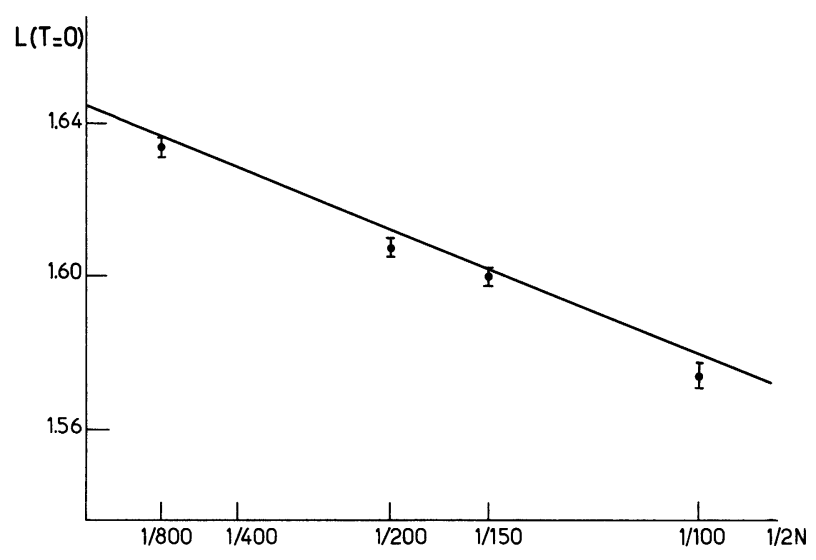

Fig. 3. - Numerical results from [14] of the length of the optimal assignment for samples with a number of points $2 N$ between 100 and 800 ; the number of samples studied is roughly $6000(2 N=100), 5000(2 N=150), 5000$ $(2 N=200), 500(2 N=800)$. The straight line is the numerical prediction (52).

\section{Remarks.}

We have shown in this paper that the spectrum of the fluctuations around the replica symmetric solutions to the matching and assignment problems contains only nonnegative eigenvalues at any temperature. Therefore these solutions are locally stable. Of course strictly speaking this is not enough to be able to assert that these are the exact solutions to the problem : we have found one saddle point which is stable but it is possible that there will exist another saddle point. This would imply a first order phase transition which seems rather unlikely. Also considering the success of the analytic prediction (52) it is quite probable that we have found the exact solutions to these two matching problems.

The formalism which we have developed for the analysis of the fluctuations allows for the full study of the stability in systems with a finite connectivity, and therefore with an infinity of order parameters at the replica symmetric level, at any temperature. One is no longer constrained to treat the fluctuations 
around the order parameters of highest order as perturbations, and finally get back to the de Almeida-Thouless formalism. We hope that this will be useful in the future in order to determine the correct solutions to dilute spin glasses, partitioning of graphs with a finite valence, or travelling salesman problem.

\section{Appendix.}

We compute the first piece of the $\mathrm{O}(1 / N)$ corrections to the free energy, $\Delta F^{1}$, which is from (8) :

$$
\begin{aligned}
\Delta F^{1}=\lim _{n \rightarrow 0} \frac{1}{4 N n \beta} \sum_{\alpha, \gamma}{ }^{\prime} g_{p(\alpha)} g_{p(\gamma)} \times \\
\times\left(\frac{Q_{p(\alpha)+p(\gamma)}^{\text {s.p. }}}{2 g_{p(\alpha)+p(\gamma)}}\right)^{2} \\
\Delta F^{1}=\frac{1}{4 N \beta} \sum_{p, q=1}^{\infty} \frac{(-1)^{p+q-1}(p+q-1) !}{p ! q !} \times \\
\times g_{p} g_{q}\left(\frac{Q_{p+q}^{\text {s.p. }}}{2 g_{p+q}}\right)^{2} .
\end{aligned}
$$

The saddle point order parameter is [3] :

$$
\frac{Q_{p+q}^{\text {s.p. }}}{2 g_{p+q}}=\int \mathrm{d} \ell \mathrm{e}^{-G(\ell)} \frac{\mathrm{e}^{\ell(p+q)}}{(p+q-1) !}
$$

and for the case of flat distances one has :

$$
\frac{g_{p} g_{q}}{g_{p+q}}=\frac{1}{\beta}\left(\frac{1}{p}+\frac{1}{q}\right) .
$$

So that :

$$
\begin{aligned}
\Delta F^{1}=\frac{1}{4 N \beta^{2}} & \sum_{p, q=1}^{\infty} \frac{(-1)^{p+q-1}}{p p ! q !} \times \\
& \times \int \mathrm{d} \ell \mathrm{e}^{-G(\ell)} Q_{p+q} \mathrm{e}^{\ell(p+q)} .
\end{aligned}
$$

We write $k=p+q$ and perform the sums over $k$ and $p$ :

$$
\begin{aligned}
\Delta F^{1}=\frac{1}{4 N \beta^{2}} \sum_{k=1}^{\infty}(-1)^{k-1} \int \mathrm{d} \ell \mathrm{e}^{-G(\ell)} \mathrm{e}^{\ell k} \frac{Q_{k}}{k !} \times \\
\quad \times \int_{0}^{1} \frac{\mathrm{d} x}{x}\left[(1+x)^{k}-1-x^{k}\right] .
\end{aligned}
$$

By the definition of the order parameter function (23), this reads :

$$
\begin{aligned}
& \Delta F^{1}=\frac{1}{4 N \beta^{2}} \int_{0}^{1} \frac{\mathrm{d} x}{x} \times \int \mathrm{d} \ell \mathrm{e}^{-G(\ell)} \times \\
& \quad \times[G(\ell+\log (1+x))-G(\ell)-G(\ell+\log x)]
\end{aligned}
$$

This formula enables one to compute numerically the change $\Delta F^{1}$ at any temperature, using the solution [3] for $G(l)$. In the zero temperature limit one knows that $G(l)$ is a function $\hat{G}(l / \beta)$ where :

$$
\hat{G}(u)=\log \left(1+\mathrm{e}^{2 u}\right) .
$$

Changing variables in (A.7) as $x=\mathrm{e}^{-\beta u}$ one gets :

$\Delta F^{1 \stackrel{T \rightarrow 0}{=}}-\frac{1}{4 N} \int_{0}^{\infty} \mathrm{d} u \int \mathrm{d} \ell \mathrm{e}^{-\hat{G}(\ell)} \hat{G}(\ell-u)$

which can be written as :

$$
\Delta F^{1 \stackrel{T \rightarrow 0}{=}}-\frac{1}{8 N} \int_{-\infty}^{+\infty} \mathrm{d} t \hat{G}(t) \hat{G}(-t)
$$

$\Delta F^{1}=-\frac{1}{4 N} \int_{0}^{\infty} \mathrm{d} t\left[(\log 2 \cosh t)^{2}-t^{2}\right]$.

The evaluation of the integral in (A.10) is not completely trivial. One method consists in noticing that :

$$
\Delta F^{1}=-\frac{1}{4 N} \frac{\mathrm{d}^{2} L}{\mathrm{~d} \nu^{2}}(\nu=0)
$$

where :

$$
L(\nu)=\int_{0}^{\infty} \mathrm{d} t\left[(2 \cosh t)^{\nu}-\mathrm{e}^{\nu t}\right]
$$

which is well defined for $\nu<2$. Now for $\nu$ negative one can use :

$$
L(-n)=\frac{1}{n}\left[\frac{\Gamma\left(1+\frac{n}{2}\right)^{2}}{\Gamma(1+n)}-1\right]
$$

Taking the second derivative of this expression with respect to $n$ at $n=0$, we get :

$$
\Delta F^{1}=-\frac{1}{N} \frac{\zeta(3)}{8}
$$

which is the announced result (10). 


\section{References}

[1] Mézard, M., Parisi, G. and Virasoro, M. A., Spin glass theory and beyond (World Scientific) 1987.

[2] For a review, see e.g. Heidelberg colloqium on glassy dynamics and optimization (Springer) 1987.

[3] Mézard, M. and Parisi, G., J. Physique Lett. 46 (1985) L-771.

[4] Orland, H., J. Physique Lett. 46 (1985) L-763.

[5] MÉzard, M. and Parisi, G., J. Physique 47 (1986) 1285.

[6] Kanter, I. and Sompolinsky, H., Phys. Rev. Lett. 58 (1987) 164.

[7] MÉzArd, M. and PARisi, G., Europhys. Lett. 3 (1987) 1067.

[8] De Dominicis, C. and Mottishaw, P., Europhys.
Lett. 3 (1987) 87 and Lecture Notes in Physics $\mathrm{n}^{\circ} 268$ (Springer-Verlag) 1987 p.123.

[9] Mézard, M., Parisi, G. and Virasoro, M. A., Europhys. Lett. 1 (1986) 77.

[10] Vannimenus, J. and Mézard, M., J. Physique Lett. 45 (1984) L-1145.

[11] Kirkpatrick, S. and Toulouse, G., J. Physique 46 (1985) 1277.

[12] De Almeida, J. R. L. and Thouless, D., J. Phys. A 11 (1978) 983.

[13] Burkard, R. E. and Derigs, U., Eds : Lectures Notes in Economics and Math Systems vol. 184 (Springer-Verlag) 1980.

[14] Brunetti, R., Krauth, W., Mézard, M. and PARISI, G., in preparation. 